\title{
Cardiac Output Measurements Based on the Pulse Wave Transit Time and Thoracic Impedance Exhibit Limited Agreement With Thermodilution Method During Orthotopic Liver Transplantation
}

\author{
Aurora Magliocca, MD, ${ }^{*} \dagger$ Emanuele Rezoagli, MD, ${ }^{*}+$ Thomas Anthony Anderson, PhD, MD,* \\ Sara Maria Burns, MS,* Fumito Ichinose, MD, PhD,* and Hovig Vatche Chitilian, MD*
}

\begin{abstract}
BACKGROUND: Orthotopic liver transplantation (OLT) is characterized by significant intraoperative hemodynamic variability. Accurate and real-time cardiac output (CO) monitoring aids clinical decision making during OLT. The purpose of this study is to compare accuracy, precision, and trending ability of $\mathrm{CO}$ estimation obtained noninvasively using pulse wave transit time (estimated continuous cardiac output [esCCO; Nihon Kohden, Tokyo, Japan]) or thoracic bioimpedance (ICON; Osypka Medical GmbH, Berlin, Germany) to thermodilution cardiac output (TDCO) measured with a pulmonary artery catheter.

METHODS: Nineteen patients undergoing OLT were enrolled. CO measurements were collected with esCCO, ICON, and thermodilution at 5 time points: (T1) pulmonary artery catheter insertion; (T2) surgical incision; (T3) portal reperfusion; (T4) hepatic arterial reperfusion; and (T5) abdominal closure. The results were analyzed with Bland-Altman plot, percentage error (the percentage of the difference between the $\mathrm{CO}$ estimated with the noninvasive monitoring device and $\mathrm{CO}$ measured with the thermodilution technique), 4-quadrant plot with concordance rate (the percentage of the total number of points in the I and III quadrant of the 4-quadrant plot), and concordance correlation coefficient (a measure of how well the pairs of observations deviate from the 45-degree line of perfect agreement).

RESULTS: Although TDCO increased at T3-T5, both esCCO and ICON failed to track the changes of $\mathrm{CO}$ with sufficient accuracy and precision. The mean bias of esCCO and ICON compared to TDCO were $-2.0 \mathrm{~L} / \min (\mathrm{SD}, \pm 2.7 \mathrm{~L} / \mathrm{min})$ and $-3.3 \mathrm{~L} / \mathrm{min}(\mathrm{SD}, \pm 2.8 \mathrm{~L} / \mathrm{min})$, respectively. The percentage error was $69 \%$ for esCCO and $77 \%$ for ICON. The concordance correlation coefficient was 0.653 (95\% confidence interval [Cl], 0.283-0.853) for esCCO and 0.310 (95\% Cl, -0.167 to 0.669$)$ for ICON. Nonetheless, esCCO and ICON exhibited reasonable trending ability of TDCO (concordance rate: $95 \%$ [95\% Cl, 88-100] and 100\% [95\% Cl, 93-100]), respectively. The mean bias was correlated with systemic vascular resistance (SVR) and arterial elastance (Ea) for esCCO (SVR, $r=0.610$, 95\% Cl, 0.216-0.833, $P<.0001$; Ea, $r=0.692$, 95\% Cl, 0.347-0.872; $P<.0001$ ) and ICON (SVR, $r=0.573,95 \% \mathrm{Cl}, 0.161-0.815, P<.0001$; Ea, $r=0.612,95 \% \mathrm{Cl}, 0.219-0.834, P<.0001$ ).

CONCLUSIONS: The noninvasive CO estimation with esCCO and ICON exhibited limited accuracy and precision, despite with reasonable trending ability, when compared to TDCO, during OLT. The inaccuracy of esCCO and ICON is especially large when SVR and Ea were decreased during the neohepatic phase. Further refinement of the technology is desirable before noninvasive techniques can replace TDCO during OLT. (Anesth Analg 2018;126:85-92)
\end{abstract}

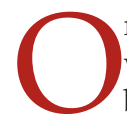
rthotopic liver transplantation (OLT) is associated with significant intraoperative hemodynamic instability secondary to changes in preload, cardiac function, and afterload. ${ }^{1-3}$ Moreover, patients with end-stage liver disease often present with elevated cardiac output (CO) and low systemic vascular resistance (SVR). ${ }^{4,5}$ In this clinical scenario, an accurate intraoperative assessment of $\mathrm{CO}$ can aid in hemodynamic management. Thermodilution technique by pulmonary artery catheter (PAC) represents the gold standard of $\mathrm{CO}$ measurement and is considered the standard intraoperative monitoring of CO in OLT. ${ }^{6}$ However, the routine use of PAC has been questioned due to its controversial impact on patient outcomes. A meta-analysis of randomized controlled trials failed to find a mortality benefit of PAC monitoring in critically ill patients. ${ }^{7}$ Furthermore, PAC is associated with an increased risk of complications. ${ }^{8-10}$
From the *Department of Anesthesia, Critical Care and Pain Medicine, Massachusetts General Hospital and Harvard Medical School, Boston Massachusetts; and †Department of Health Science, School of Medicine and Surgery, University of Milan-Bicocca, Milan, Italy.

Accepted for publication March 27, 2017

Funding: This study was supported by a Sponsored Research Agreement from Nihon Kohden.

Conflicts of Interest: See Disclosures at the end of the article.

Supplemental digital content is available for this article. Direct URL citations appear in the printed text and are provided in the HTML and PDF versions of this article on the journal's website (www.anesthesia-analgesia.org).
A. Magliocca and E. Rezoagli contributed equally to this work; F. Ichinose and H. V. Chitilian contributed equally to this work.

Reprints will not be available from the authors.

Address correspondence to Fumito Ichinose, MD, PhD, Department of Anesthesia, Critical Care and Pain Medicine, Massachusetts General Hospital, Harvard Medical School, 149 13th St, CNY 149, Room 4315, Charlestown, MA 02129. Address e-mail to fichinose@mgh. harvard.edu.

Copyright (C 2017 International Anesthesia Research Society

DOI: $10.1213 /$ ANE.0000000000002171 
A number of devices have been developed to monitor $\mathrm{CO}$ noninvasively, including estimated continuous cardiac output (esCCO; Nihon Kohden, Tokyo, Japan) and ICON (Osypka Medical GmbH, Berlin, Germany). The esCCO technology is based on the pulse wave transit time (PWTT), the time between electrocardiogram R-wave peak and the pulse oximeter plethysmograph upstroke. PWTT negatively correlates with esCCO-calculated stroke volume. ${ }^{11}$ The ICON estimates CO by measuring the changes of thoracic electrical impedance. ${ }^{12}$ The accuracy of esCCO and ICON, compared to thermodilution cardiac output (TDCO), has been evaluated in clinical studies, both in surgical and in intensive care unit patients. ${ }^{13-18}$ The results are equivocal, requiring further assessment of these new techniques. One of the most challenging clinical settings where $\mathrm{CO}$ is routinely monitored is liver transplantation surgery. Indeed, OLT is characterized by large intravascular volume shifts and marked changes in vascular tone. ${ }^{3}$

The purpose of this study is to evaluate accuracy, precision of agreement, and the trending ability of $\mathrm{CO}$ measured with 2 noninvasive devices (esCCO and ICON) compared to TDCO with PAC, in patients undergoing OLT.

\section{METHODS}

This prospective, method comparison study was conducted at the Massachusetts General Hospital (Boston, MA) after obtaining approval from the Partners Healthcare Institutional Review Board. This article adheres to the Strengthen the Reporting of Observational studies in Epidemiology (STROBE) statement. ${ }^{19}$ After obtaining verbal consent, 19 patients undergoing OLT were enrolled. The inclusion criteria were age $>18$ years old and the intraoperative use of a PAC. Exclusion criteria were the presence of acute liver failure, severe pulmonary disease, severe cardiac disease, persistent arrhythmias, and body weight $<40 \mathrm{~kg}$.

\section{Hemodynamic Monitoring and Invasive Measurements of Cardiac Output With Pulmonary Artery Catheter}

All patients were monitored in accordance with the American Society of Anesthesiology standards. ${ }^{20}$ Intra-arterial blood pressure monitoring was conducted with radial arterial catheters. Based on the anesthesiologist's preference, transesophageal echocardiography was also used in some patients. Following the induction of general anesthesia, a PAC (Swan-Ganz Thermodilution AV Paceport Catheter; Edwards Life Sciences, Irvine, CA) was placed via an introducer in the right internal jugular vein (2 Lumen Central Venous Kit with 9Fr Access Sheath; Arrow International, Reading, PA). TDCO measurements were performed by injecting $10 \mathrm{~mL}$ isotonic solution at operating room temperature $\left(65^{\circ} \mathrm{F}\right)$ into the central venous port of the PAC within 4 seconds. At each predetermined time point, TDCO was recorded as the average of 3 measurements that were within $15 \%$ of each other. If the difference between the TDCO measurements exceeded $15 \%$, the injections were repeated to obtain a set of 3 TDCO measurements within 5 minutes.

\section{Noninvasive Measurements of Cardiac Output With esCCO and ICON}

To measure $\mathrm{CO}$ by esCCO, 3 additional noninvasive electrocardiogram leads and 1 dedicated additional pulse oximeter finger probe were applied to the patient. The esCCO system calculates $\mathrm{CO}$ according to the formula esCCO $=K \times(\alpha \times$ PWTT $+\beta) \times H R$, where $K$ quantifies arterial compliance: $K=\mathrm{SV} / \mathrm{PP}$ (SV = stroke volume, $\mathrm{PP}=$ pulse pressure), $\alpha$ and $\beta$ are experimental constants, and $\mathrm{HR}$ is heart rate. The estimated $\mathrm{CO}$ were displayed on the Nihon Kohden BSM-9101 bedside monitor (Nihon Kohden) and continuously recorded during all the surgical interventions. The esCCO system and the BSM-9101 bedside monitor were provided by Nihon Kohden. Although the esCCO technology has been officially approved and used in 68 countries and regions including European Union, South Korea, Brazil, and Russia, it has not been approved by the US FDA for noninvasive measurements of $\mathrm{CO}$ at the time of writing.

The ICON device was connected to the patient via the application of 4 additional electrocardiogram leads. ICON estimates $\mathrm{CO}$ by measuring the variations of thoracic electrical impedance. ${ }^{12}$ esCCO and ICON were then calibrated, after entering the biometric data (age, sex, height, weight, plus a measurement of blood pressure for esCCO). Although one can calibrate $\mathrm{CO}$ estimation with esCCO against TDCO at the initiation of the measurements, we did not calibrate esCCO using TDCO in the current study to determine the ability of esCCO to estimate $\mathrm{CO}$ independent of PAC. The ICON device was provided by Osypka Medical.

With regard to the timing of the $\mathrm{CO}$ measurements, concurrent with the display of $\mathrm{CO}$ value by thermodilution after saline injection, $\mathrm{CO}$ values of esCCO and ICON values were recorded. An average of $3 \mathrm{CO}$ measurements for both esCCO and ICON was considered at each time point.

$\mathrm{CO}$ measurements were performed by all 3 techniques during 5 intraoperative time points: (1) following PAC insertion (T1); (2) immediately following surgical incision (T2); (3) at portal reperfusion (T3); (4) at hepatic arterial reperfusion (T4); and (5) at the start of the abdominal closure (T5). At each time point, hemodynamic data were collected: systolic (SAP), diastolic (DAP), and mean (MAP) arterial pressure; HR; central venous pressure (CVP); systolic, diastolic, and mean pulmonary artery pressure. SVR $\left[S V R=80 \cdot(\mathrm{MAP}-\mathrm{CVP}) \cdot \mathrm{TDCO}^{-1}\right]$ and arterial elastance $[\mathrm{Ea}$ $\left.=(2 \cdot \mathrm{SAP}+\mathrm{DAP}) \cdot(3 \cdot \mathrm{SV})^{-1}\right]$ were calculated. The intraoperative hemodynamic and medical management was at the discretion of the attending anesthesiologist, without taking into account the $\mathrm{CO}$ measurements performed by the 2 study devices.

\section{Statistical Analysis}

Patient characteristics are expressed as mean \pm SD, median (25\%-75\% percentile) or count and proportion. Normality of data was assessed using the Shapiro-Wilk test. All $P$ values were 2 sided, and $P<.05$ was considered statistically significant. When applicable, $P$ values were appropriately adjusted for multiple testing. All statistical analyses were performed using $\mathrm{R}$ statistical software (RStudio, version 3.2.2; R Foundation for Statistical Computing, Vienna, Austria), GraphPad Prism 6 for Mac OS $\times$ version 6.0f (GraphPad Software Inc, La Jolla, CA), and Microsoft Excel for Mac 2011 version 14.4.7 (Microsoft Corporation, Redmond, WA). 


\section{Precision of TDCO, esCCO, and ICON}

The CO precision error $(\%)$ and the least significant change $(\%)$ were calculated for TDCO, esCCO, and ICON at each time point, as previously reported. ${ }^{16}$

\section{Hemodynamic Measures}

The pathophysiology of OLT is characterized by a profound hemodynamic variability during surgery. To describe the relevant hemodynamic change over time of such a surgical procedure, repeated measurements 1-way analysis of variance with Bonferroni correction or Friedman test with Dunn multiple comparisons test was used to examine differences at each time point versus T1 (PAC insertion), as appropriate. This analysis was applied to all the reported hemodynamic variables (HR, SAP, DAP, MAP, CVP, systolic pulmonary artery pressure, diastolic pulmonary artery pressure, mean pulmonary artery pressure, SVR, $\mathrm{Ea}$, and the $\mathrm{CO}$ measured with thermodilution, ICON, and es(CO).

To examine differences between $\mathrm{CO}$ device measurements, comparisons between TDCO, esCCO, and ICON were evaluated at each time point using the 1-way analysis of variance with Bonferroni correction or Kruskal-Wallis test with post hoc Dunn multiple comparison testing, according to the data distribution.

\section{Regiression Analyses}

Scatter plots of CO measurements for TDCO versus esCCO and TDCO versus ICON were performed at each time point. Linear regression analyses was performed, and Pearson correlation coefficients $(r)$ with $95 \%$ confidence interval (CI) were reported. Linear regression analyses including all time points for TDCO versus esCCO and TDCO versus ICON were performed and correlation coefficients were corrected for repeated measurements. ${ }^{21}$

\section{Agreement Analysis}

The agreement between esCCO and ICON with TDCO measurements was assessed using the Bland-Altman method..$^{22}$ The Bland-Altman analysis is essential to determine information on the accuracy and precision of esCCO and ICON. Accuracy was evaluated studying the Bland-Altman bias. Precision was assessed examining: (1) The limits of agreement of the Bland-Altman bias, which was calculated as $\pm 1.96 \times$ SD bias; (2) The $95 \%$ CI of the upper and lower limits of agreement of the Bland-Altman bias, which was calculated as $\pm 1.96 \times \sqrt{ }\left(3 \times s 2 / n^{\prime}\right)$, where $s$ was the $S D$ of the measurement differences between the 2 methods, and $n^{\prime}$ was the adjusted sample size. ${ }^{22}$ The adjusted sample size is calculated utilizing the equation $n^{\prime}=n(1+p(m-1))$, where $\mathrm{n}$ is the sample size, $\mathrm{p}$ is the intraclass correlation coefficient (ICC), and $\mathrm{m}$ is the number of measures per subject. To account for the repeated observations within each subject, the Bland-Altman approach for repeated measures was applied when calculating the bias, standard deviation, and limits of agreement. ${ }^{23}$ The percentage error (PE) was calculated as $95 \%$ limit of agreement $(1.96 \times \mathrm{SD}$ from the bias) divided by the mean $\mathrm{CO}$ (calculated as the mean of tested device and TDCO) times 100 . A PE of $<30 \%$ is suggested to be acceptable for clinical use. ${ }^{24}$

\section{Trending Ability}

To evaluate the ability to track changes in $\mathrm{CO}$ (trending ability) of these devices, we performed a 4-quadrant plot analysis and calculated the linear regression of the $\mathrm{CO}$ change with ICON and esCCO versus the change with TDCO, as well as the concordance correlation coefficient (CCC), both accounting for repeated measurements..$^{21,25,26}$ The linear regression correlation coefficients are calculated through an analysis of variance approach which incorporates the sum of squares of the subject-level variation. ${ }^{21}$ For the CCC calculation, the R package "cccrm" is utilized, which includes the appropriate ICC as an estimator of the $\mathrm{CCC}$ and uses the variance components from a linear mixed model. ${ }^{26}$ Changes in $\mathrm{CO}$ values in the 4-quadrant plot were calculated by subtracting $\mathrm{CO}$ values at subsequent time points and implementing an exclusion zone of $0.75 \mathrm{~L} / \mathrm{min}$, which is considered noise. ${ }^{16}$ We reported the concordance rate (CR) among the changes $(\Delta)$ of $C O$ between the 5 time points for ICON and esCCO versus TDCO, which was calculated according to the 4-quadrant plot (proportion of data points in which both methods demonstrate change of $\mathrm{CO}$ in the same direction). The point estimates and CI for concordance were calculated using a random intercepts model with subject ID being treated as a random effect. To measure the precision $(\mathrm{r})$ and accuracy $\left(\mathrm{C}_{\mathrm{b}}\right)$ of the trending ability, the CCC $\left(C C C=r \times C_{b}\right)$ was also calculated, accounting for repeated measurements and utilizing the variance components from a linear mixed model. The appropriate ICC was used to estimate the CCC through the R package "ccrm." ${ }^{25,26}$ The CCC ranges from 0 to 1 with higher values indicating stronger agreement.

\section{Sample Size}

We used the repeated measurements Bland-Altman approach to estimate the mean bias and the limits of agreement around bias. ${ }^{22,23}$ Although no a priori sample size considerations were made, based on precisions, enrolling 19 participants who are each measured 5 times produces an expected precision of $\pm 1.33 \mathrm{~L} / \mathrm{min}$ for the limits of agreement while comparing esCCO and TDCO, and an expected precision of $\pm 1.53 \mathrm{~L} / \mathrm{min}$ for the limits of agreement while comparing ICON and TDCO.

\section{RESULTS}

We enrolled 19 patients undergoing OLT in this prospective, method comparison study. Clinical and surgical characteristics of the patients are shown in Table 1. Random error of each technology was reported in Supplemental Digital Content 1, Table, http://links.lww.com/AA/B781. Hemodynamic measurements at each time point are presented in Table 2. As shown, there were no significant differences in CVP, MAP, and mPAP over time, while HR significantly increased at T5 (T5 versus T1, $P<.05$ ). Compared to T1, SVR was significantly reduced at $\mathrm{T} 4$ and $\mathrm{T} 5$ (T4 versus $\mathrm{T} 1, P<.05$; $\mathrm{T} 5$ versus $\mathrm{T} 1, P<.01)$ and Ea significantly decreased at T4 and T5 (T4 versus $\mathrm{T} 1, P<.05 ; \mathrm{T} 5$ versus $\mathrm{T} 1, P<.05)$. Intraoperative $\mathrm{CO}$ measurements with TDCO, esCCO, and ICON are shown in Figure 1, Supplemental Digital Content 2, Figure 1, http:/ / links.lww.com/AA/B782, and Table 2. TDCO significantly increased at T3 (portal reperfusion) and T4 (hepatic arterial 
reperfusion) with a peak of 50\% increase at T5 (abdominal closure) compared to T1 (T3 versus T1, $P<.01$; T4 versus $\mathrm{T} 1, P<.01 ; \mathrm{T} 5$ versus T1, $P<.01)$. In contrast, neither esCCO nor ICON showed significant differences in $\mathrm{CO}$ measures

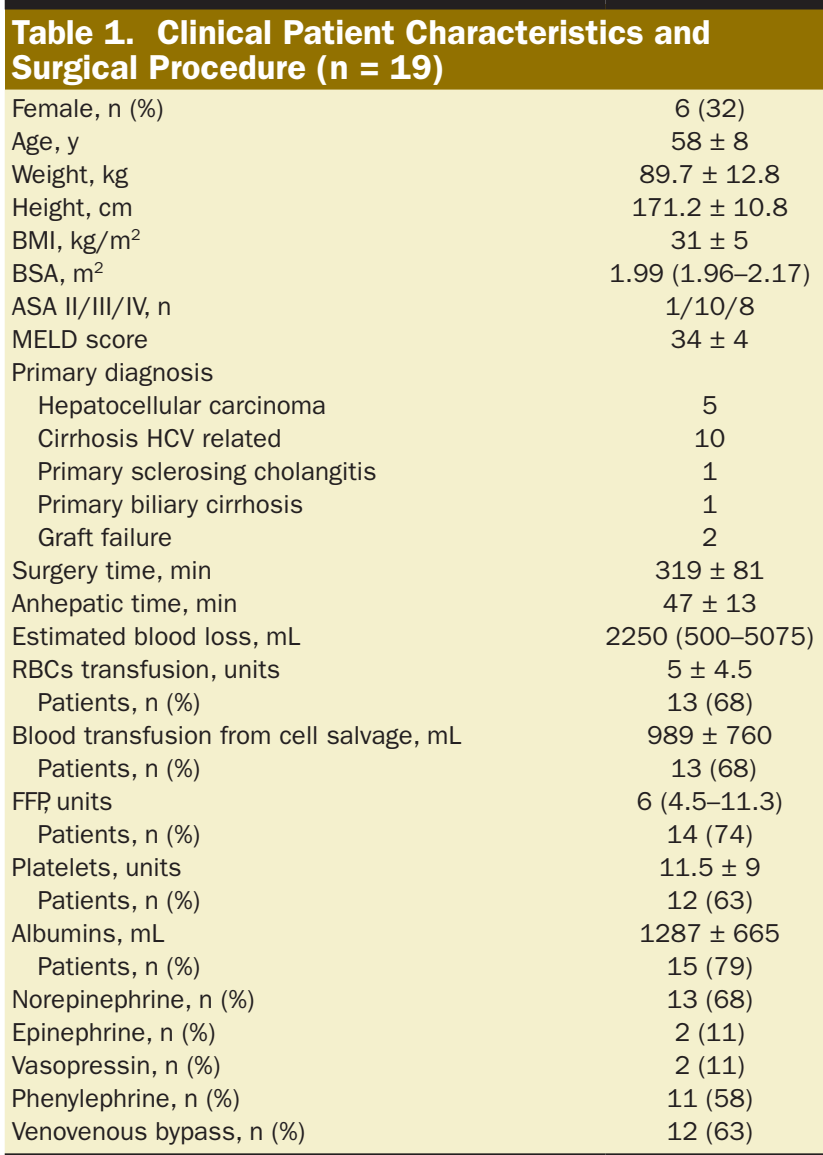

Data are expressed as mean \pm SD, median and interquartile ranges (25\%$75 \%$ percentile) or count (\%), as appropriate.

Abbreviations: ASA, American Society of Anesthesiologists physical status classification system; BMI, body mass index; BSA, body surface area; FFP, fresh frozen plasma; HCV, hepatitis C virus; MELD, model of end score liver disease; RBCs, red blood cells. during the entire surgical procedure. esCCO and ICON were compared to TDCO per time point. esCCO was significant lower at T3 $(P<.01)$, T4 $(P<.01)$, and T5 $(P<.001)$, while ICON was significantly lower than TDCO at all time points, even at baseline $(\mathrm{T} 1, P<.01 ; \mathrm{T} 2, P<.001 ; \mathrm{T} 3, P<.001$; T4, $P<.001 ;$ T5, $P<.0001)$.

\section{Regression Analyses}

Single time point scatter plots for TDCO versus esCCO (Supplemental Digital Content 3, Figure 2A-E, http:// links.lww.com/AA/B783) and TDCO versus ICON (Supplemental Digital Content 4, Figure 3A-E, http:// links.lww.com/AA/B784) were assessed. Linear regression analyses corrected for repeated measurements including all the time points (T1-T5) showed moderately strong correlation between TDCO and esCCO $(r=0.733,95 \%$ CI, $0.418-$ 0.890, $P<.0001$; Supplemental Digital Content 3, Figure 2F, http://links.lww.com/AA/B783), as well as between TDCO and ICON $(r=0.659,95 \%$ CI, 0.293-0.856, $P<.0001$; Supplemental Digital Content 4, Figure 3F, http://links. lww.com/AA/B784).

\section{Accuracy and Precision of Agreement}

Bland-Altman analyses were performed considering all the time points (T1-T5) and corrected for repeated measurements (19 measurements repeated 5 times). The bias between esCCO and TDCO was $-2.0 \mathrm{~L} / \mathrm{min}$, and the SD bias was $\pm 2.7 \mathrm{~L} / \mathrm{min}$; $95 \% \mathrm{CI}$ of the limits of agreement ranged from lower $-7.4 \pm 1.3 \mathrm{~L} / \mathrm{min}$ to upper $3.3 \pm 1.3 \mathrm{~L} /$ min (Figure 2A). The bias between ICON and TDCO was $-3.3 \mathrm{~L} / \mathrm{min}$, and the SD bias was $\pm 2.8 \mathrm{~L} / \mathrm{min}$; $95 \% \mathrm{CI}$ of the limits of agreement ranged from lower $-8.8 \pm 1.5 \mathrm{~L} / \mathrm{min}$ to upper $2.2 \pm 1.5 \mathrm{~L} / \mathrm{min}$ (Figure $2 \mathrm{~B}$ ). A bias estimate close to zero with a narrow CI can be interpreted as highly precise and accurate agreement between 2 measurements. The esCCO and ICON devices are reporting lower CO measurements than the TDCO standard, and the CIs reveal about a 5 to $6 \mathrm{~L} / \mathrm{min}$ range in the precision of these measurements. PEs of esCCO and ICON were $69 \%$ and $77 \%$, respectively.

\begin{tabular}{|c|c|c|c|c|c|}
\hline & T1 & T2 & T3 & T4 & T5 \\
\hline HR (beats/min) & $63 \pm 10$ & $70 \pm 16$ & $74 \pm 14$ & $73 \pm 14$ & $77 \pm 14^{a}$ \\
\hline SAP (mm Hg) & $117(108-130)$ & $127(101-139)$ & $122(114-135)$ & $129(105-142)$ & $127(108-134)$ \\
\hline DAP (mm Hg) & $61 \pm 12$ & $64 \pm 14$ & $62 \pm 8$ & $62 \pm 11$ & $61 \pm 12$ \\
\hline MAP (mm Hg) & $84 \pm 17$ & $88 \pm 18$ & $87 \pm 9$ & $86 \pm 14$ & $82 \pm 13$ \\
\hline CVP $\left(\mathrm{cm} \mathrm{H} \mathrm{H}_{2} \mathrm{O}\right)$ & $11(9-16)$ & $12(8-14)$ & $9(6-11)$ & $9(6-12)$ & $12(6-15)$ \\
\hline $\mathrm{sPAP}(\mathrm{mm} \mathrm{Hg})$ & $34(30-40)$ & $33(27-38)$ & $31(27-37)$ & $31(26-38)$ & $34(28-43)$ \\
\hline $\mathrm{dPAP}(\mathrm{mm} \mathrm{Hg})$ & $14(11-18)$ & $15(10-18)$ & $12(6-14)$ & $10(8-13)$ & 14 (9-19) \\
\hline mPAP (mm Hg) & $24(20-28)$ & $23(18-27)$ & $18(15-25)$ & $18(16-24)$ & $24(18-30)$ \\
\hline SVR (dyn.s.cm- $\mathrm{cm}^{-5}$ ) & $940(613-1143)$ & $644(580-1217)$ & 746 (501-901) & $604(509-844)^{a}$ & $535(429-751)^{b}$ \\
\hline $\mathrm{Ea}(\mathrm{mm} \mathrm{Hg} / \mathrm{mL})$ & $1 \pm 0.3$ & $1 \pm 0.4$ & $0.9 \pm 0.2$ & $0.8 \pm 0.2^{\mathrm{a}}$ & $0.8 \pm 0.2^{\mathrm{a}}$ \\
\hline TDCO (L/min) & $5.5(4.6-8.4)$ & $7.3(5.6-9.4)$ & $9.6(7-11.6)^{b}$ & $10.4(7-11.5)^{b}$ & $11(7.4-11.8)^{b}$ \\
\hline $\operatorname{ICON}(\mathrm{L} / \mathrm{min})$ & $3.5(2.9-5.2)^{c}$ & $4.4(3.9-5.9)^{d}$ & $5.6(3.8-8.7)^{d}$ & $5.4(4-9.1)^{d}$ & $5.4(3.9-6.3)^{e}$ \\
\hline $\operatorname{escco}(\mathrm{L} / \mathrm{min})$ & $5.2(4.3-6.3)$ & $5.7(5.1-6.7)$ & $7.1(5-8.9)^{c}$ & $6.6(4.5-8.9)^{c}$ & $6.8(5.6-9.2)^{d}$ \\
\hline
\end{tabular}

Data are expressed as mean \pm SD or median and interquartile ranges (25\%-75\% percentile), as appropriate.

Abbreviations: CVP, central venous pressure; DAP, diastolic arterial pressure; dPAP, diastolic pulmonary artery pressure; Ea, arterial elastance; esCCO, estimated continuous cardiac output; HR, heart rate; MAP, mean arterial pressure; mPAP, mean pulmonary artery pressure; SAP, systolic arterial pressure; sPAP, systolic pulmonary artery pressure; SVR, systemic vascular resistance; TDCO, thermodilution cardiac output.

a $P<.05,{ }^{b} P<.01$ versus $\mathrm{T} 1$.

${ }^{\mathrm{c}} P<.01,{ }^{\mathrm{d}} P<.001,{ }^{\mathrm{e}} P<.0001$ versus TDCO. 


\section{Trending Ability}

The trending ability of these 2 devices is expressed by the 4-quadrant plot and the CCC (Figure 3). An exclusion zone of $0.75 \mathrm{~L} / \mathrm{min}$ was applied, according to the literature. ${ }^{16}$ There was a strong correlation between $\triangle \mathrm{esCCO}$ and $\Delta \mathrm{TDCO}(P<.0001, r=0.775,95 \% \mathrm{CI}, 0.496-0.909)$ and moderate correlation between $\triangle \mathrm{ICON}$ and $\triangle \mathrm{TDCO}(P<.0001$, $r=0.445,95 \%$ CI, -0.011 to 0.748 ; Figure 3).

The CCC between $\triangle \mathrm{esCCO}$ and $\triangle \mathrm{TDCO}$ was $0.653(95 \%$ CI, 0.283-0.853), whereas the CCC between $\triangle \mathrm{ICON}$ and $\triangle \mathrm{TDCO}$ was only 0.310 (95\% CI, -0.167 to 0.669$)$. The CR was $95 \%$ (95\% CI, $88-100)$ between $\triangle \mathrm{esCCO}$ and $\triangle \mathrm{TDCO}$ and $100 \%(95 \%$ CI, 93-100) between $\triangle \mathrm{ICON}$ and $\triangle \mathrm{TDCO}$ (Figure 3). While the CCCs are modest, the CRs are very high, indicating good trending ability in both the esCCO and the ICON devices. As shown in Figure 1, the direction of agreement is consistent even though the accuracy and precision are mediocre.

\section{SVR, Ea, and Bias}

The bias of noninvasive $\mathrm{CO}$ measurement devices (esCCO and ICON) was correlated with SVR (esCCO: $P<.0001$, $r=0.610,95 \%$ CI, 0.216-0.833; ICON: $P<.0001, r=0.573$,

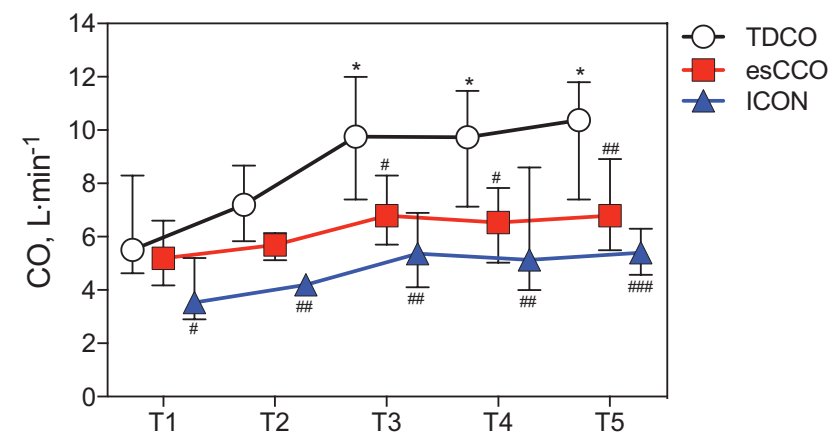

Figure 1. Intraoperative cardiac output (CO) measurements with the thermodilution technique (thermodilution cardiac output [TDCO]; circles, black line), estimated continuous cardiac output (esCCO; squares, red line), and electrical cardiometry (ICON; triangles, blue line). Data are presented as median with 95\% confidence interval. T1, insertion of pulmonary artery catheter; T2, surgical incision; T3, portal venous reperfusion; T4, hepatic arterial reperfusion; T5, start of abdominal closure. ${ }^{*} P<.01$, versus T1. $\# P<.01$, \#\#P<.001, and \#\#\#P $<.0001$ versus TDCO.
95\% CI, 0.161-0.815). When SVR decreased, the absolute value of Bland-Altman bias increased for both devices (Figure 4A and 4B). To assess the vascular load, the Ea was calculated. There was a strong correlation between the bias of noninvasive $\mathrm{CO}$ and Ea in both devices (esCCO: $P<.0001$, $r=0.692,95 \%$ CI, 0.347-0.872; ICON: $P<.0001, r=0.612$, $95 \%$ CI, 0.219-0.834). The absolute value of Bland-Altman bias increased as Ea decreased (Figure 4C and 4D). These results suggest that systemic vasodilation and increased systemic arterial compliance might worsen the accuracy of esCCO and ICON.

\section{DISCUSSION}

In the current study, we observed that $\mathrm{CO}$ measurements with the noninvasive $\mathrm{CO}$ monitors, esCCO and ICON, were neither accurate nor precise compared to TDCO during OLT. However, both esCCO and ICON showed good trending ability of TDCO. esCCO had a smaller bias (more accuracy) and narrower limits of agreement (more precision) than ICON. The ability of esCCO and ICON to track variations of $\mathrm{CO}$ was particularly poor during conditions of low SVR and high CO. Both SVR and Ea closely correlated with the bias of esCCO and ICON compared to TDCO.

Although the PEs of both esCCO and ICON were greater than $30 \%$, as proposed by Critchley and Critchley, ${ }^{24}$ esCCO showed a lower PE than ICON ( $48 \%$ vs $60 \%$, respectively). These results are comparable with previous studies comparing esCCO to thermodilution. ${ }^{13,27,28}$ Results on the comparison between ICON and TDCO are conflicting. Some authors reported that ICON was accurate in critically ill patients. ${ }^{18}$ On the other hand, Critchley et $\mathrm{al}^{29}$ showed that ICON was not accurate when changes in peripheral resistance occurred, consistent with the current observations.

The 4-quadrant plot analysis revealed similar CRs of the 2 tested devices; $95 \%$ for esCCO and $100 \%$ for ICON, greater than the accepted minimum limit of $92 \%$, when exclusion zone $>0.75 \mathrm{~L} / \mathrm{min}$ was applied. ${ }^{16}$ These findings suggest that these monitors exhibit high trending ability. To assess not only accuracy but also precision of the techniques, we used CCCs. The CR only explores if the direction of change of the $\mathrm{CO}$ calculated by the study devices is in agreement with the change of the $\mathrm{CO}$ measured by TDCO. Instead, the CCC includes a measure of the deviation of the best-fit
Figure 2. Bland-Altman plots for repeated measurements between cardiac output (CO) measurements obtained with estimated continuous cardiac output (esCCO) and thermodilution cardiac output (TDCO; red squares; $\mathrm{A})$, and electrical cardiometry (ICON) and TDCO (blue triangles; B). The continuous lines correspond to the mean difference (bias), the dashed lines correspond to the $95 \%$ limits of agreement (LA), and the dotted lines correspond to the $95 \%$ confidence interval of the upper and lower LA. PE indicates percentage error.
A

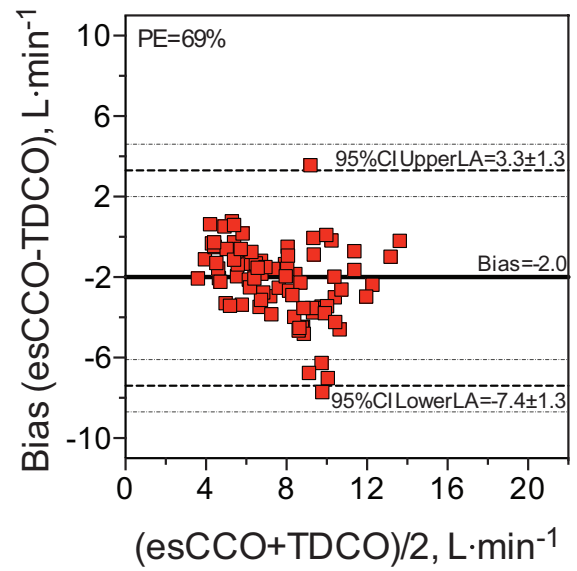

B

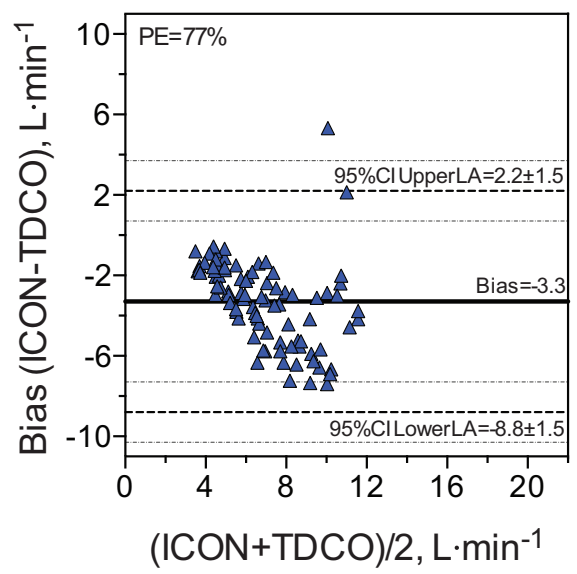



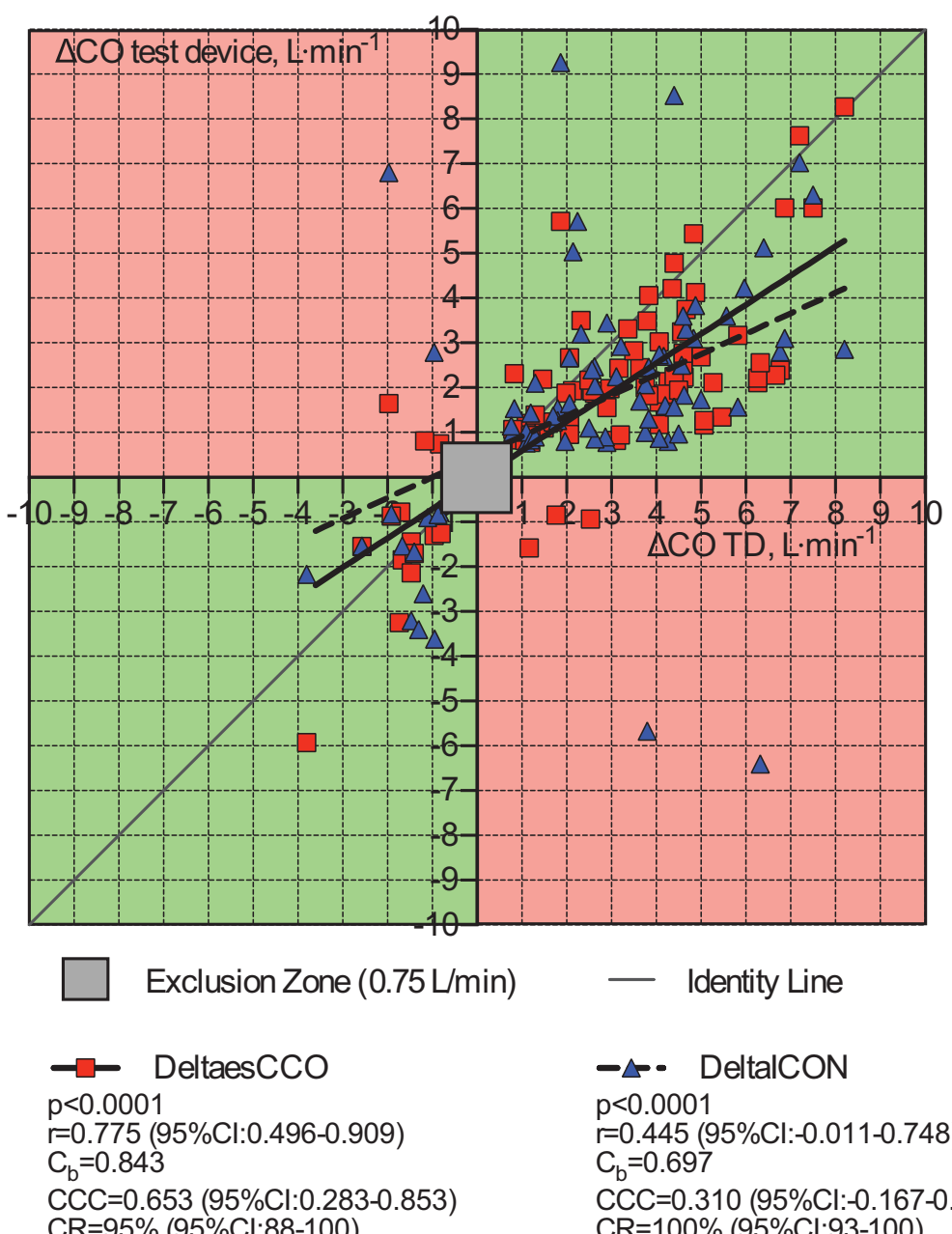

- Identity Line

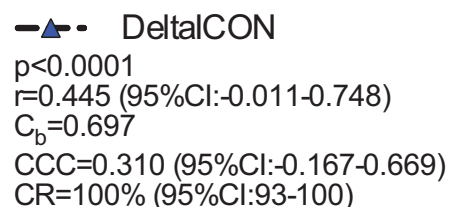

Figure 3. Four-quadrant plot corrected for repeated measurements shows changes in cardiac output ( $\mathrm{CO}$ ) with estimated continuous cardiac output (esCCO; $\triangle$ esCCO, red squares) and with electrical cardiometry (ICON; $\triangle \mathrm{ICON}$; blue triangles) compared to changes in thermodilution $\mathrm{CO}(\triangle \mathrm{TDCO})$. Exclusion zone, set at $0.75 \mathrm{~L} /$ $\min$, is shown. Regression lines for $\triangle \mathrm{esCCO}$ (continuous black line) and $\triangle \mathrm{ICON}$ (dashed line) and the identity line (continuous dark gray line) are shown. In light green, concordant CO changes. In salmon, discordant $\mathrm{CO}$ changes. CCC indicates concordance correlation coefficient; $\mathrm{Cl}$, confidence interval; $\mathrm{CR}$, concordance rate. line from the identity line. We found that CCC for esCCO (0.653) and ICON (0.310) were lower than 0.9 (Figure 3), which means a poor agreement between the 2 techniques and TDCO, as suggested by McBride. ${ }^{30}$

Only few previous published studies assessed the trending ability of these techniques. In patients undergoing partial hepatectomy, esCCO was found to provide an acceptable CR of $96 \% .{ }^{15}$ However, these authors used an exclusion zone $<0.5 \mathrm{~L} / \mathrm{min}$ in the 4 -quadrant plot analysis, and they calibrated esCCO against TDCO. In the current study, we decided not to calibrate the esCCO system invasively with TDCO. While calibration of esCCO with TDCO is likely to increase the accuracy of esCCO, we sought to determine the reliability of esCCO independently of TDCO.

Our data showed that in a hyperdynamic state with uncoupling of flow-pressure relationship (ie, low SVR, with high $\mathrm{CO}$ ), esCCO failed to accurately estimate CO compared to the TDCO. Indeed, the significant correlation between bias and SVR $(P<.0001, r=0.610)$ provided an explanation of $\mathrm{CO}$ underestimation (Figure 4A). Previous studies reported an increased esCCO bias at low SVR in intensive care unit and critically ill patients. ${ }^{14,17}$ We calculated the Ea as $\left[\mathrm{Ea}=(2 \times \mathrm{SAP}+\mathrm{DAP}) \times(3 \times \mathrm{SV})^{-1}\right] \cdot{ }^{31}$ Ea describes the ability to accomplish the stroke volume and is the reciprocal of compliance. esCCO estimates the vascular compliance $\left(K=\mathrm{SV} \times \mathrm{PP}^{-1} ; \mathrm{SV}=\right.$ stroke volume; $\mathrm{PP}=$ pulse pressure $)$ at the time of calibration, using another measurement system (usually TDCO). When the calibration against TDCO is omitted, as in the current study, the esCCO calculates $K$ from the patient demographic data, using the formula obtained from a multiple regression analysis. ${ }^{32}$ The latter was performed with patient data derived from the multicenter study of Yamada et al. ${ }^{13}$ Of note, height and weight of patient population in the study by Yamada et $\mathrm{al}^{13}$ were significantly smaller than those of patients in the current study (mean $\Delta$ height $=-11 \mathrm{~cm}$, mean $\Delta$ weight $=-24 \mathrm{~kg}$ ), casting a doubt about the appropriateness of the $K$ value used in the current study. After calibration, the fixed $K$ value is utilized to estimate the $\mathrm{CO}$ according to the formula $\mathrm{CO}=\mathrm{K} \times(\alpha \times \mathrm{PWTT}+\beta) \times$ $\mathrm{HR}$ in esCCO throughout the case. Our results showed that when Ea decreased significantly, esCCO failed to estimate $\mathrm{CO}$ accurately. Indeed, we found a significant negative correlation between esCCO bias and Ea. The absolute value of bias increased as Ea decreased (Figure 4C). It seems possible that accuracy of estimated $\mathrm{CO}$ by esCCO increases if the $K$ value is adjusted in the course of measurements.

Similarly, in the ICON system, which is bioimpedance based, changes in SVR or Ea can profoundly affect the CO estimation. Indeed, ICON is based on the assumption that the volumetric aortic enlargement during systole is directly related to the blood flow, regardless of the influence of other factors. ${ }^{29}$ When SVR or Ea is significantly decreased, it is 
A

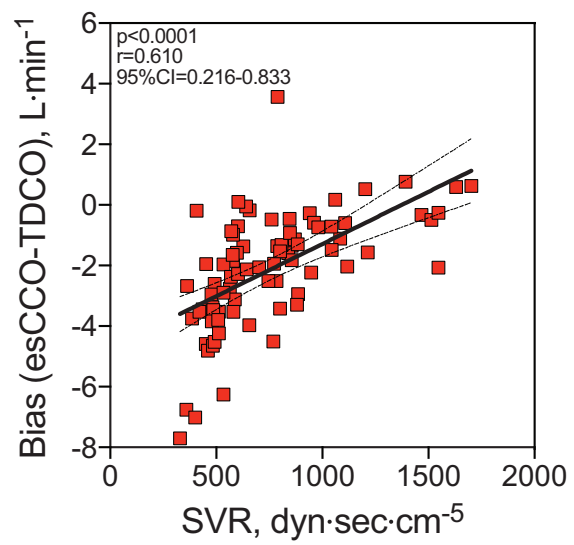

Figure 4. Linear regression between bias and systemic vascular resistance (SVR) for estimated (A) continuous cardiac output (esCCO; red squares) and (B) ICON (blue triangles); (C) linear regression between bias and arteria elastance (Ea) for esCCO (red squares) and (D) ICON (blue triangles). $\mathrm{Cl}$ indicates confidence interval; TDCO, thermodilution cardiac output.

C
B
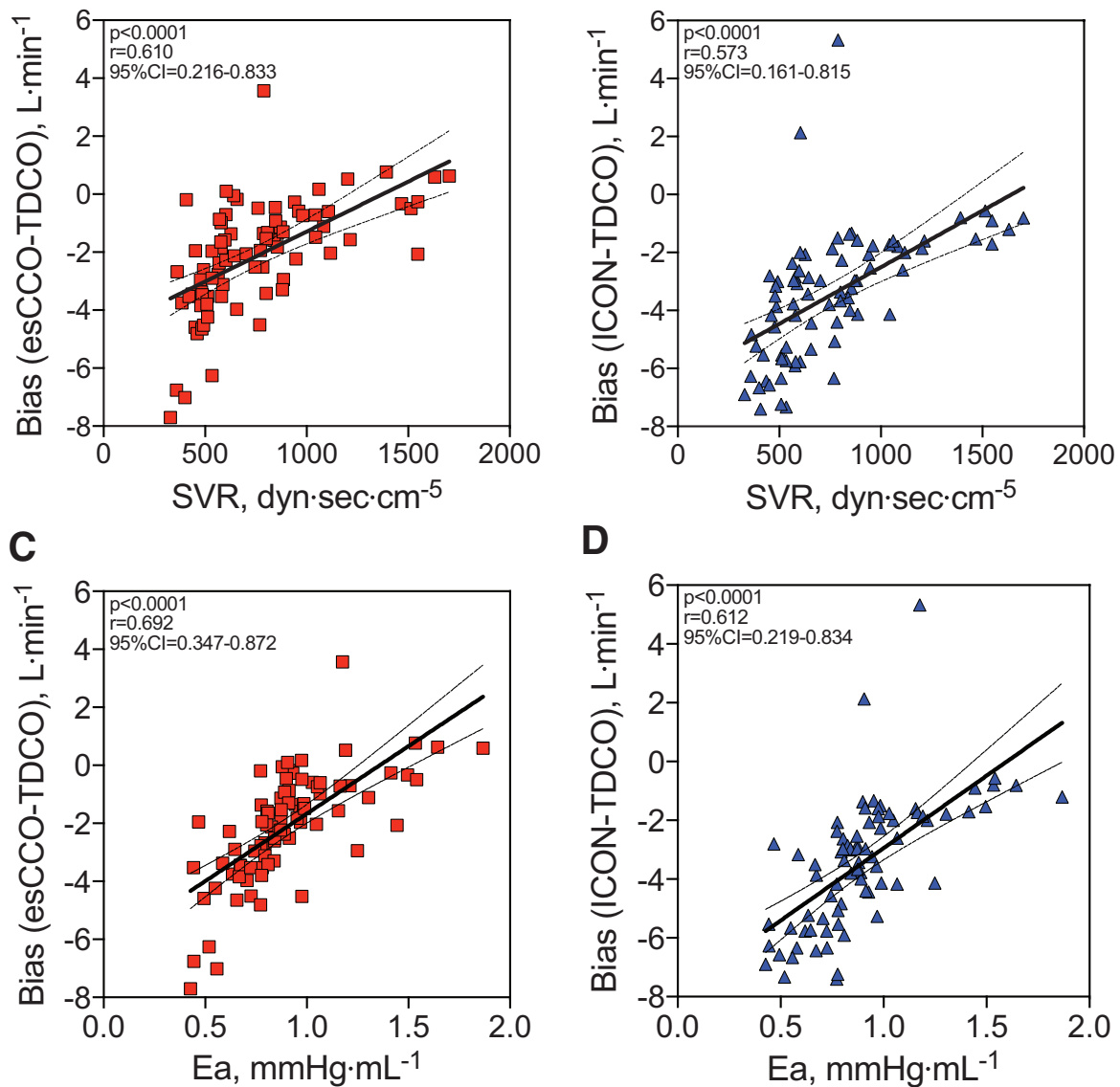

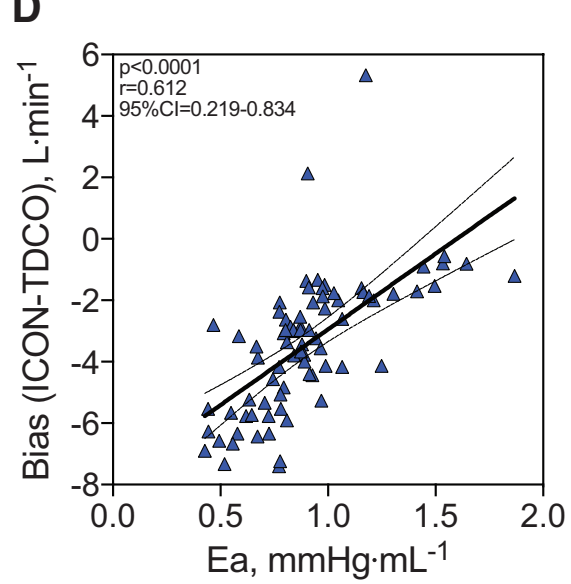

likely that the relationship between the aortic enlargement and systolic blood flow will change. Accordingly, we found that the bias of ICON from TDCO became greater when SVR and Ea decreased (Figure 4B and 4D).

Our study has some limitations. The sample size is small because we focused on patients undergoing OLT in a single hospital. While our patient population was relatively homogeneous (Table 1), it is still uncertain whether or not our finding is applicable to other patients undergoing OLT especially at other institutions. Although we found that the accuracy and precision of esCCO and ICON are limited in patients undergoing OLT, it is possible that these noninvasive $\mathrm{CO}$ monitors are more reliable when used in less demanding clinical scenarios where hemodynamic swing is minimum. ${ }^{33}$ However, we chose to examine these devices in the cases with the highest acuity when continuous $\mathrm{CO}$ monitoring will be most useful to clinicians and limitation of their reliability is likely to be exposed.

In conclusion, the noninvasive $\mathrm{CO}$ estimation with esCCO and ICON exhibited limited accuracy and precision, despite with reasonable trending ability, when compared to TDCO during OLT. esCCO is more accurate and precise than ICON, but both of them exhibited insufficient agreement with TDCO. These results suggest that both techniques are not clinically interchangeable with thermodilution-based CO measurements during OLT. Our results also suggest that the drastic change in SVR and Ea might play a contributing role on the inaccuracy of the esCCO and
ICON. The refinement of the current technologies focusing on the inclusion of SVR or Ea changes in the CO calculation may be warranted, to make noninvasive $\mathrm{CO}$ estimation more reliable during surgery characterized by profound intraoperative hemodynamic instability.

\section{ACKNOWLEDGMENTS}

Authors thank Osypka Medical for generously providing an ICON monitor. Osypka Medical provided no financial support and had no role in the data collection or analysis.

\section{DISCLOSURES}

Name: Aurora Magliocca, MD.

Contribution: This author helped design and perform experiments, analyze and interpret data, and write the manuscript.

Conflicts of Interest: None.

Name: Emanuele Rezoagli, MD.

Contribution: This author helped analyze and interpret data and write the manuscript.

Conflicts of Interest: None.

Name: Thomas Anthony Anderson, PhD, MD

Contribution: This author helped perform experiments and revise and edit the manuscript.

Conflicts of Interest: None.

Name: Sara Maria Burns, MS

Contribution: This author helped analyze and interpret data, and revise and edit the manuscript.

Conflicts of Interest: None.

Name: Fumito Ichinose, MD, PhD.

Contribution: This author helped conceive the project, design experiments, interpret data, and write the manuscript. 
Conflicts of Interest: F. Ichinose serves as a Consultant and an Advisory Board Member of Nihon Kohden Innovation Center (a subsidiary of Nihon Kohden).

Name: Hovig V. Chitilian, MD.

Contribution: This author helped design and perform experiments, provide important intellectual input, and revise and edit the manuscript.

Conflicts of Interest: None.

This manuscript was handled by: Maxime Cannesson, MD, PhD.

\section{REFERENCES}

1. De Wolf AM. 6/2/06 Perioperative assessment of the cardiovascular system in ESLD and transplantation. Int Anesthesiol Clin. 2006;44:59-78.

2. Hall TH, Dhir A. Anesthesia for liver transplantation. Semin Cardiothorac Vasc Anesth. 2013;17:180-194.

3. Paugam-Burtz C, Kavafyan J, Merckx P, et al. Postreperfusion syndrome during liver transplantation for cirrhosis: outcome and predictors. Liver Transpl. 2009;15:522-529.

4. Kowalski HJ, Abelmann WH. The cardiac output at rest in Laennec's cirrhosis. J Clin Invest. 1953;32:1025-1033.

5. Murray JF, Dawson AM, Sherlock S. Circulatory changes in chronic liver disease. Am J Med. 1958;24:358-367.

6. Schumann R, Mandell MS, Mercaldo N, et al. Anesthesia for liver transplantation in United States academic centers: intraoperative practice. J Clin Anesth. 2013;25:542-550.

7. Shah MR, Hasselblad V, Stevenson LW, et al. Impact of the pulmonary artery catheter in critically ill patients: meta-analysis of randomized clinical trials. JAMA. 2005;294:1664-1670.

8. Richard C, Warszawski J, Anguel N, et al; French Pulmonary Artery Catheter Study Group. Early use of the pulmonary artery catheter and outcomes in patients with shock and acute respiratory distress syndrome: a randomized controlled trial. JAMA. 2003;290:2713-2720.

9. Sandham JD, Hull RD, Brant RF, et al; Canadian Critical Care Clinical Trials Group. A randomized, controlled trial of the use of pulmonary-artery catheters in high-risk surgical patients. $N$ Engl J Med. 2003;348:5-14.

10. Harvey S, Harrison DA, Singer M, et al; PAC-Man study collaboration. Assessment of the clinical effectiveness of pulmonary artery catheters in management of patients in intensive care (PACMan): a randomised controlled trial. Lancet. 2005;366:472-477.

11. Sugo Y, Ukawa T, Takeda S, Ishihara H, Kazama T, Takeda J. A novel continuous cardiac output monitor based on pulse wave transit time. Conf Proc IEEE Eng Med Biol Soc. 2010;2010:2853-2856.

12. de Sitter A, Verdaasdonk RM, Faes TJ. Do mathematical model studies settle the controversy on the origin of cardiac synchronous trans-thoracic electrical impedance variations? A systematic review. Physiol Meas. 2016;37:R88-R108.

13. Yamada T, Tsutsui M, Sugo $Y$, et al. Multicenter study verifying a method of noninvasive continuous cardiac output measurement using pulse wave transit time: a comparison with intermittent bolus thermodilution cardiac output. Anesth Analg. 2012;115:82-87.

14. Bataille B, Bertuit M, Mora M, et al. Comparison of esCCO and transthoracic echocardiography for non-invasive measurement of cardiac output intensive care. Br J Anaesth. 2012;109:879-886.

15. Tsutsui M, Araki $\mathrm{Y}$, Masui $\mathrm{K}$, et al. Pulse wave transit time measurements of cardiac output in patients undergoing partial hepatectomy: a comparison of the esCCO system with thermodilution. Anesth Analg. 2013;117:1307-1312.

16. Thonnerieux M, Alexander B, Binet C, Obadia JF, Bastien $\mathrm{O}$, Desebbe O. The ability of esCCO and ECOM monitors to measure trends in cardiac output during alveolar recruitment maneuver after cardiac surgery: a comparison with the pulmonary thermodilution method. Anesth Analg. 2015;121:383-391.

17. Biais M, Berthezène R, Petit L, Cottenceau V, Sztark F. Ability of esCCO to track changes in cardiac output. $\mathrm{Br} J$ Anaesth. 2015;115:403-410.

18. Zoremba N, Bickenbach J, Krauss B, Rossaint R, Kuhlen R, Schälte G. Comparison of electrical velocimetry and thermodilution techniques for the measurement of cardiac output. Acta Anaesthesiol Scand. 2007;51:1314-1319.

19. Knottnerus A, Tugwell P. STROBE-a checklist to Strengthen the Reporting of Observational Studies in Epidemiology. J Clin Epidemiol. 2008;61:323.

20. American Society of Anesthesiologists Committee on Standards and Practice Parameters. Standard for basic anesthetic monitoring. Available at: http://www.asahq.org/ /media/Sites/ ASAHQ/Files/Public/Resources/standards-guidelines/standards-for-basic-anesthetic-monitoring.pdf. Accessed January 31, 2017.

21. Bland JM, Altman DG. Calculating correlation coefficients with repeated observations: Part 1-Correlation within subjects. BMJ. 1995;310:446.

22. Bland JM, Altman DG. Statistical methods for assessing agreement between two methods of clinical measurement. Lancet. 1986;1:307-310.

23. Bland JM, Altman DG. Agreement between methods of measurement with multiple observations per individual. J Biopharm Stat. 2007;17:571-582.

24. Critchley LA, Critchley JA. A meta-analysis of studies using bias and precision statistics to compare cardiac output measurement techniques. J Clin Monit Comput. 1999;15:85-91.

25. Carrasco JL, Jover L. Estimating the generalized concordance correlation coefficient through variance components. Biometrics. 2003;59:849-858.

26. Carrasco JL, Martinez JP. Concordance correlation coefficient for repeated (and non-repeated) measures. Available at: https:/ / cran.r-project.org/web/packages/cccrm/cccrm.pdf. Accessed January 31, 2017.

27. Sinha AC, Singh PM, Grewal N, Aman M, Dubowitz G. Comparison between continuous non-invasive estimated cardiac output by pulse wave transit time and thermodilution method. Ann Card Anaesth. 2014;17:273-277.

28. Ball TR, Tricinella AP, Kimbrough BA, et al. Accuracy of noninvasive estimated continuous cardiac output (esCCO) compared to thermodilution cardiac output: a pilot study in cardiac patients. J Cardiothorac Vasc Anesth. 2013;27:1128-1132.

29. Critchley LA, Peng ZY, Fok BS, James AE. The effect of peripheral resistance on impedance cardiography measurements in the anesthetized dog. Anesth Analg. 2005;100:1708-1712.

30. McBride GB. A proposal for strength-of-agreement criteria for Lin's concordance correlation coefficient. NIWA Client Report HAM 2005-062. Hamilton, New Zealand: National Institute of Water and Atmospheric Research Ltd, May 2005.

31. Kelly RP, Ting CT, Yang TM, et al. Effective arterial elastance as index of arterial vascular load in humans. Circulation. 1992;86:513-521.

32. Ishihara H, Sugo $Y$, Tsutsui M, et al. The ability of a new continuous cardiac output monitor to measure trends in cardiac output following implementation of a patient information calibration and an automated exclusion algorithm. J Clin Monit Comput. 2012;26:465-471.

33. Cannesson M, Manach YL. Noninvasive hemodynamic monitoring: no high heels on the farm; no clogs to the opera. Anesthesiology. 2012;117:937-939. 